\title{
Mycobacterium tuberculosis as a Cause of Periprosthetic Joint Infection After Total Knee Arthroplasty: A Review of the Literature
}

\author{
Andrew S. Bi ${ }^{1}$, Daniel $\mathrm{Li}^{1}$, Yunlong $\mathrm{Ma}^{2}$, Decheng $\mathrm{Wu}^{2}$, Yuangzheng $\mathrm{Ma}^{2}$ \\ 1. Orthopaedics, Northwestern University Feinberg School of Medicine, Chicago, USA 2. Orthopaedics, Second \\ Affiliated Hospital of Guangzhou Medical University, Guangzhou, CHN
}

Corresponding author: Andrew S. Bi, andrew.bi@uconn.edu

\begin{abstract}
Total knee arthroplasty (TKA) has become one of the most popular and successful surgeries performed in the world. Infection remains one of the most dreaded complications following TKA, and while rare, tuberculosis as a microbial etiology remains difficult to both diagnose and treat. A review was performed using PubMed, the Cochrane Database of Systematic Reviews, and EMBASE to identify literature pertinent to Mycobacterium tuberculosis infection, TKAs, periprosthetic joint infections, and any combination of the three. The diagnosis of tuberculosis infection after TKA is difficult due to nonspecific signs and symptoms and diagnostic testing. The surgeon should use a comprehensive approach to incorporate the patient's medical history, physical exam, and blood and imaging diagnostics. Among these, bacterial culture and histopathological examination remain the gold standard of diagnosis, but Polymerase chain reaction technology offers another, more sensitive and rapid option. Treatment strategy centers around on the cornerstone of anti-tuberculosis medical therapy and surgery depending on the clinical situation. While there is a lack of primary literature and standardized guidelines for the diagnosis and treatment of tuberculosis infection after TKA, the overarching principles of the treatment of tuberculosis and the treatment of the periprosthetic infection can be implemented together. There remains room for original research and improvements in both diagnostic testing and treatment.
\end{abstract}

Received 03/06/2019

Review began 03/07/2019 Review ended 03/22/2019 Published 03/26/2019

๑) Copyright 2019

$\mathrm{Bi}$ et al. This is an open access article distributed under the terms of the Creative Commons Attribution License CC-BY 3.0., which permits unrestricted use, distribution, and reproduction in any medium, provided the original author and source are credited.
Categories: Infectious Disease, Orthopedics, Public Health

Keywords: mycoplasma tuberculosis, tuberculosis (tb), revision total knee arthroplasty, total knee arthroplasty, periprosthetic infection, postoperative infection

\section{Introduction And Background}

With increasing life expectancy in both developed and underdeveloped countries, the incidence and prevalence of knee osteoarthritis have been steadily increasing [1-3]. In the United States alone, the number of primary total knee arthroplasties (TKAs) is projected to increase by 673\%, approximately 3.48 million operations per year [4]. In addition, the average age of surgery continues to decrease, with the demand for primary TKAs in patients less than 65 years old encompassing over $50 \%$ of all TKAs [5]. This rise can be attributed to the vast success of the primary TKA, widely considered as one of the most effective procedures of modern surgery [6]. However, a multitude of complications still exist, including but not limited to: resistant pain, hardware loosening, periprosthetic fracture, venous thromboembolisms (VTEs) and infection [7-8].

Infection, in particular, is a dreaded and severe complication after knee replacement surgery. Infection is the most common cause of revision TKA, as the indication for $25.2 \%$ of all revision TKAs [9]. The vast majority of infections are caused by typical bacterial organisms, such as Staphylococcus aureus, or Staphylococcus epidermidis; however, Mycobacterium tuberculosis (TB) is a known inciting bacterium that has not been extensively studied. Tuberculosis periprosthetic joint infection (TBPJI) poses a significant burden on the healthcare system, with an average length of hospital stay of 5.1-7.6 days and an average cost of USD 49,360 [9]. Infection not only impacts the patient and the healthcare system, but it also has a drastic effect on surgeons' own emotions [10]. While the majority of TBPJIs occur within the first two years after surgery, approximately $25 \%$ of all TBPJIs occur after two years from primary procedure [11].

First reported by Wolfgang et al. [12] in 1978, TBPJI continues to lack guidelines for diagnosis and treatment. There are many difficulties with early diagnosis of TBPJI, and there are currently no established standards for treatment. The goal of this article is to review and summarize the current body of literature in order to provide a framework for the clinical diagnosis and treatment of TBPJI after TKA.

\section{Review}

\section{Etiology}

The cause of postoperative tuberculosis infection can be attributed to one of the two factors: patient factors 
and operative factors [13]. Patient factors revolve primarily around comorbidities that contribute to an immunosuppressed state: advanced age, obesity, diabetes, autoimmune disorders, large burns, chronic steroid therapy, immunomodulating medications, or retroviral infections [14-16]. The surgery itself can cause an inflammatory response that can both reactivate an old or latent tuberculosis infection, as well as reduce the ability of the immune system to fight off infection.

Postoperative knee joint tuberculosis can be divided into three sources: (1) recurrence of previous tuberculosis infection in a native or postoperative knee; (2) dissemination of tuberculosis from the original source in the lungs or extrapulmonary source; and (3) reactivation of a latent tuberculosis infection [17].

\section{Diagnosis}

The diagnosis of tuberculosis infection after TKA is difficult. Harwin et al. [17] reviewed the literature and divided tuberculosis infection after TKA into early and late onset. Early-onset infections were classified as those occurring less than eight weeks after surgery, while late-onset infections were classified as those occurring more than eight weeks post-op. The difficulty of diagnosis originates from the lack of specific clinical symptoms and timely laboratory tests, which often results in delayed diagnosis [18]. Tokumoto et al. [19] believe that there are three reasons for the delayed diagnosis of tuberculosis infection after TKA: (1) a missed diagnosis of joint tuberculosis infection before TKA, (2) suspected tuberculosis infections are difficult to diagnose, and (3) other, more readily diagnosable bacterial infections that can misdirect clinical management. In addition, due to the low pre-test probability of TBPJI, the atypical nature of the presentation, and frequent lack of minor criteria, classic definitions of prosthetic joint infections, such as that of the Musculoskeletal Infection Society (MSIS), can result in false negatives [20]. Currently, the gold standard for diagnosis includes a joint fluid or synovial tissue analysis for acid-fast bacilli culture and histopathological examination [18]. Although the diagnostic specificity is not high, surgeons must consider the patient's medical history, clinical presentation, as well as imaging findings when approaching their medical decision making.

\section{Medical History}

Patients with underlying causes of immunocompromise, history of tuberculosis infection, or patients with tuberculosis risk factors should consider the possibility of postoperative tuberculosis infection prophylaxis [13]. Marschall et al. [21] reported about a 48-year-old male patient with a history of HIV and CD4+ count of $7 / \mathrm{uL}$, who presented with painless swelling of the joint six months after TKA. He was diagnosed with TBPII at nine months and passed away one month after diagnosis. The tuberculosis was found to be disseminated throughout multiple organ systems.

Local or Systemic Symptoms and Signs

When a TKA is complicated by TBPJI, local symptoms of the knee are mostly inflammatory reactions such as pain, swelling, and fever. More severe signs can manifest in a small proportion of the population, such as purulent drainage, abscess or sinus formation. Harwin et al. [17] reported on a 60-year-old female patient who developed a $4 \mathrm{~cm}$ by $4 \mathrm{~cm}$ painful mass on the medial side of the proximal metatarsal, seven months after TKA. The mass gradually enlarged, and surgical exploration demonstrated a cheese-like granulation tissue on the inside of the mass.

In patients with local tuberculosis infection after TKA, the classic systemic symptoms of tuberculosis infection such as weight loss, fatigue, or night sweats are rare. In these patients, the only other presenting symptom besides local symptoms of TBPJI can be a second local infection, i.e. of the lungs, or a second joint. Wang et al. [22] reported on a 72-year-old male patient with a 50-year smoking history who developed a chronic cough and progressive knee pain one year following a TKA. Sputum cultures and knee synovial tissue culture confirmed the diagnosis to be TBPJI and pulmonary tuberculosis infection. Bryan et al. [23] reported about the case of a 72-year-old female with knee pain and swelling after TKA, along with a painful, swollen elbow. She denied any history of tuberculosis infection, yet histological examination found caseous granulomas, confirming the diagnosis. Other complications secondary to contiguous spread can present, such as local bone destruction, osteomyelitis, and prosthetic loosening.

\section{Blood Diagnostic Testing}

Laboratory tests from plasma for TBPJI have extremely low specificity [24]. Oftentimes laboratory results can be within normal reference ranges. When abnormalities do result, they usually include an elevated white blood cell count, or increased inflammatory markers, such as erythrocyte sedimentation rate and C-reactive protein. However, these hematologic indicators have poor specificity, acting as markers of general inflammatory activity rather than indicating the patient has an infection by Mycobacterium tuberculosis [25]. One blood test of note would be the QuantiFERON-TB Gold ${ }^{\circledR}$, which is an interferon-gamma release assay (IGRA). However, due to the limitation of measuring a surrogate marker of tuberculosis, it cannot distinguish between latent and active infection, and thus its usefulness in the acute setting is limited [26]. 
Acid-fast bacilli (AFB) staining and cultures, along with a histopathological examination of synovial tissue demonstrating caseating granulomas remains the gold standard for the diagnosis of Mycobacterium tuberculosis [27]. However, even with these methods, there can be false negatives in testing. If the surgeon's tissue biopsy misses an area of active infection, or if a bacterial load is too low to culture on LöwensteinJensen medium, false negatives can result. In addition, the growth of tuberculosis in cultures often takes weeks, with an average of 23.7 days [28]. Besser et al. [29] reported on a primary TKA case for osteoarthritis, in which synovial hyperplasia and erythema were observed during operation. Biopsy of the tissue returned positive for tuberculosis infection. Marmor et al. [30] reported that a 66-year-old male developed knee pain and swelling two months after right TKA. Doppler ultrasonography showed an abscess in the right popliteal fossa and was subsequently surgically removed and cultured. While the results of the joint and abscess cultures were negative, the AFB were finally isolated from blood samples on acid-fast staining and culture. An example of a case that emphasizes the importance of multiple tissue samples comes from Wray et al. [31], in which diagnosis of tuberculosis infection was made not with direct tissue biopsy, but rather sputum culture in conjunction with clinical symptoms, allowing a 63-year-old male to receive early directed therapy for TBPJI.

Polymerase Chain Reaction Technology

Polymerase chain reaction (PCR) technology has recently changed the paradigm for the diagnosis of tuberculosis. It has the advantages of rapid turnaround, increased sensitivity, and ability to detect trace loads of bacteria, causing a higher percentage of diagnoses to be made with PCR in recent years [24]. Some studies have shown PCR can even detect tuberculosis infection from tissue samples of purulent material or granulation tissue in TBPJI [32]. In testing respiratory samples, sensitivity and specificity are $87.1 \%$ and $99.9 \%$, respectively, and when testing extrapulmonary specimens, sensitivities range from $53.7 \%$ to $100 \%$ depending on sample selection. Neogi et al. [33] reported on a 73-year-old female who developed TBPJI 14 years after a TKA with negative synovial tissue and joint fluid cultures. Ultimately, PCR of synovial tissue detected Mycobacterium tuberculosis.

Imaging

Imaging for the diagnosis of tuberculosis infection is often focused around the lungs, with chest radiographs and non-contrast computed tomography (CT) scans the primary focus for active vs. latent infection [34]. Imaging for extrapulmonary tuberculosis has been less extensively studied. Imaging findings in musculoskeletal tuberculosis infection, in particular, are often nonspecific and only indicate a non-specific infectious etiology [35]. In the initial stages of the disease, plain radiographs can show widening of the joint space with soft tissue swelling. With further disease progression, marginal erosions, blurring of the articular cortex, and loss of subchondral bone can be seen [35]. A classic constellation of findings was described in Phemister's triad: juxta-articular osteoporosis, peripherally located osseous erosions, and gradual narrowing of the joint space [36]. The use of CT and magnetic resonance imaging (MRI) is limited secondary to artifact produced from the metal implant, a problem not unique to TBPJI. Nuclear medicine techniques have been found to be sensitive but not specific [34-35]. Zeiger et al. [37] and Spinner et al. [38] reported on the use of radionuclide scanning to show that the knee joint of surgical sites had increased absorption of tracer (99mTc-MDP, etc.), but was not specific to the diagnosis of tuberculosis infection.

\section{Treatment strategy}

There are currently no established standards in managing patients with TBPJI. Key principles are source control, anti-tuberculosis medications, and early diagnosis and treatment, or empiric therapy in cases when a diagnosis might be delayed. Wray et al. [31], reported on a case in which, after developing fever and knee pain following a primary TKA, a 63-year-old male patient was empirically put on anti-tuberculosis treatment. Histopathologic diagnosis followed a few weeks later, and a year after surgery the knee joint had recovered without the need for revision surgery. However, in most cases, the general treatment paradigm involves anti-tuberculosis drug therapy in addition to a thorough joint debridement. Depending on the timing and severity of infection, the prosthesis can occasionally be preserved, with revision surgery and fusion providing alternative options [17].

\section{Anti-tuberculosis Medical Therapy}

According to the Infectious Disease Society of America (IDSA), recommended medical treatment regimens for extrapulmonary tuberculosis are similar to the pulmonary disease. This consists of initiation of two months of "RIPE" therapy; rifampin, isoniazid, pyrazinamide, and ethambutol, followed by six to nine months of rifampin and isoniazid [39]. On occasions when TBPJI occurs early, within six to eight weeks, standard anti-tuberculosis medical therapy alone is possible. However, this treatment regimen requires not only rapid diagnosis, but also no other sites of infection, prosthetic loosening, or osteolysis. There are scant reports of late-onset TBPJIs being successfully treated with medical therapy, but the majority of current literature supports surgical intervention in addition to anti-tuberculosis treatment [40]. 
The option of treating TBPJI with I\&D and medical therapy while retaining the prosthesis is mainly limited to the following clinical situations: (1) early-onset infection; (2) clinical and imaging findings consistent with an absence of prosthetic loosening; and (3) sensitivity to anti-tuberculosis medications [41]. Multiple case reports have demonstrated success in treating the infected joint with extensive lavage and antibiotics $[29,31]$. These all cases included a diagnosis within eight weeks following primary surgery, and an extended course of medical therapy greater than nine months, allowing the original prosthesis to be preserved.

\section{Anti-tuberculosis Medical Therapy, Joint I\&D, and Two-Stage Replacement Arthroplasty}

Nearly all late-onset TBPJI, severe suppurative infections, active sinus tract formation, signs of periprosthetic osteolysis or prosthetic loosening, should undergo a two-stage replacement arthroplasty, as results have been shown to be as effective as $90 \%$ [42]. Patients must be medically fit for multiple surgeries and have adequate bone stock, but this two-stage revision remains the gold standard for a late-onset joint infection following arthroplasty [43]. Spinner et al. [38] reported on a 70-year-old female who had recurrent suppurative sinus tract formation following a primary TKA. She was later diagnosed with tuberculosis infection, and after multiple debridements, underwent a two-stage revision along with anti-tuberculosis medications for 12 months. The patient did well, without a need for further revision or fusion. Khater et al. [44] reported on a 75-year-old female that was found to have a $2-3 \mathrm{~mm}$ wound in the surgical incision site three months after a right primary TKA. Histopathologic testing revealed tuberculosis infection, and a twostage revision was performed using vancomycin and rifampin spacers. After 18 months of anti-tuberculosis medications, there was no recurrence of TBPJI. These are just a few examples of successful cases of treatment of late-onset TBPJI.

Anti-tuberculosis Treatment, Removal of Prosthesis and Arthrodesis

Knee arthrodesis is a procedure resulting in severe limitation of functionality and quality of life. Nearly all arthrodeses are performed secondary to failed knee arthroplasties, with the most common indications due to PJI, extensor mechanism disruption, soft-tissue deficiency, and severe bone loss [45-47]. Carrega et al. [48] reported on multiple patients who developed recurrent TBPJI after primary TKA and subsequent revision surgeries. Biopsy of infected tissue confirmed tuberculosis infection, and following debridement, the patients underwent arthrodesis and extensive courses of anti-tuberculosis drug therapy. There were no recurrences, and the patients were able to ambulate a year after surgery. While arthrodesis is not a first-line treatment option, it can be a useful option for patients with persistent infection and medical comorbidities limiting the ability to tolerate multiple procedures [49].

\section{Conclusions}

TB is a rare etiology of PJI after primary TKA, and diagnosis requires a high level of pretest suspicion along with a knowledge of the various non-specific signs and symptoms. Bacterial AFB culture and histopathological examination continue to be the current gold standard for diagnosis; however, modern PCR technology has improved to become a useful tool in the surgeon's arsenal for the diagnosis of TBPJI. There is a vast room for future research to improve the standardization and systemization of the diagnosis paradigm in attempts to improve not only the sensitivity and specificity but also the speed and rapidity of diagnosis. The current treatment regimen continues to center around the classic anti-tuberculosis initial quadruple therapy. Medical therapy alone has rare and limited indications and is relegated primarily to mild cases of early-onset post-operative infections. The key treatment divergence centers around retention or replacement of the original prosthesis. Often times prosthetic aseptic loosening or persistent infection are the core factors which influence the decision-making process. For late-onset infections, the current gold standard of treatment is similar to those of other PJIs with more common microbes. Two-stage revision arthroplasty can eradicate the infection and provide higher quality functional outcomes, but in difficult cases, arthrodesis or even amputation is occasionally necessary. The exact choice of treatment for each patient should be specifically tailored to the clinical scenario, but future work needs to be done in analyzing quantitative outcome data following the various types of treatment. Due to the lack of current literature, case reports and case series continue to be a useful source of primary data and should be encouraged to be published with standardized reporting with a focus on thorough patient demographics and evidence-based outcome data.

\section{Additional Information \\ Disclosures}

Conflicts of interest: In compliance with the ICMJE uniform disclosure form, all authors declare the following: Payment/services info: All authors have declared that no financial support was received from any organization for the submitted work. Financial relationships: All authors have declared that they have no financial relationships at present or within the previous three years with any organizations that might have an interest in the submitted work. Other relationships: All authors have declared that there are no other relationships or activities that could appear to have influenced the submitted work. 


\section{References}

1. Maradit KH, Larson DR, Crowson CS, et al.: Prevalence of total hip and knee replacement in the United States. J Bone Joint Surg Am. 2015, 97:1386-1397. 10.2106/JBJS.N.01141

2. Jonsson H, Olafsdottir S, Sigurdardottir S, et al.: Incidence and prevalence of total joint replacements due to osteoarthritis in the elderly: risk factors and factors associated with late life prevalence in the AGESReykjavik Study. BMC Musculoskelet Disord. 2016, 17:14. 10.1186/s12891-016-0864-7

3. Kawata M, Sasabuchi Y, Inui H, et al.: Annual trends in knee arthroplasty and tibial osteotomy: Analysis of a national database in Japan. Knee. 2017, 24:1198-1205. 10.1016/j.knee.2017.06.005

4. Kurtz S, Ong K, Lau E, Mowat F, Halpern M: Projections of primary and revision hip and knee arthroplasty in the United States from 2005 to 2030. J Bone Joint Surg Am. 2007, 89:780-785. 10.2106/JBJS.F.00222

5. Kurtz SM, Lau E, Ong K, Zhao K, Kelly M, Bozic KJ: Future young patient demand for primary and revision joint replacement: national projections from 2010 to 2030. Clin Orthop Relat Res. 2009, 467:2606-2612. 10.1007/s11999-009-0834-6

6. Meding JB, Meding LK, Ritter MA, Keating EM: Pain relief and functional improvement remain 20 years after knee arthroplasty. Clin Orthop Relat Res. 2012, 470:144-149. 10.1007/s11999-011-2123-4

7. Martin JR, Beahrs TR, Stuhlman CR, Trousdale RT: Complex primary total knee arthroplasty: long-term outcomes. J Bone Joint Surg Am. 2016, 98:1459-1470. 10.2106/JBJS.15.01173

8. Baldini A, Castellani L, Traverso F, Balatri A, Balato G, Franceschini V: The difficult primary total knee arthroplasty: a review. Bone Joint J. 2015, 97:30-39. 10.1302/0301-620X.97B10.36920

9. Bozic KJ, Kurtz SM, Lau E, et al.: The epidemiology of revision total knee arthroplasty in the United States . Clin Orthop Relat Res. 2010, 468:45-51. 10.1007/s11999-009-0945-0

10. Mallon C, Gooberman-Hill R, Blom A, Whitehouse M, Moore A: Surgeons are deeply affected when patients are diagnosed with prosthetic joint infection. PLoS ONE. 2018, 13:e0207260. 10.1371/journal.pone.0207260

11. Kurtz SM, Ong KL, Lau E, Bozic KJ, Berry D, Parvizi J: Prosthetic joint infection risk after TKA in the Medicare population. Clin Orthop Relat Res. 2010, 468:52-56. 10.1007/s11999-009-1013-5

12. Wolfgang GL: Tuberculosis joint infection. Clin Orthop Relat Res. 1978, 136:257-263.

13. Kim S-J, Kim JH: Late onset Mycobacterium tuberculosis infection after total knee arthroplasty: a systematic review and pooled analysis. Scand J Infect Dis. 2013, 45:907-914. 10.3109/00365548.2013.830192

14. Salt E, Wiggins AT, Rayens MK, et al.: Moderating effects of immunosuppressive medications and risk factors for post-operative joint infection following total joint arthroplasty in patients with rheumatoid arthritis or osteoarthritis. Semin Arthritis Rheum. 2017, 46:423-429. 10.1016/j.semarthrit.2016.08.011

15. Porrett PM, Hashmi SK, Shaked A: Immunosuppression: trends and tolerance?. Clin Liver Dis. 2014, 18:687716. 10.1016/j.cld.2014.05.012

16. Brouard S, Budde K: Current status of immunosuppressive minimization and tolerance strategies . Transpl Int. 2015, 28:889-890. 10.1111/tri.12624

17. Harwin SF, Banerjee S, Issa K, Kapadia BH, Pivec R, Khanuja HS, Mont MA: Tubercular prosthetic knee joint infection. Orthopedics. 2013, 36:e1464-e1469. 10.3928/01477447-20131021-35

18. Garg SK, Tiwari RP, Tiwari D, et al.: Diagnosis of tuberculosis: available technologies, limitations, and possibilities. J Clin Lab Anal. 2003, 17:155-163. 10.1002/jcla.10086

19. Tokumoto JI, Follansbee SE, Jacobs RA: Prosthetic joint infection due to Mycobacterium tuberculosis: report of three cases. Clin Infect Dis. 1995, 21:134-136. 10.1093/clinids/21.1.134

20. Parvizi J, Zmistowski B, Berbari EF, et al.: New definition for periprosthetic joint infection: from the Workgroup of the Musculoskeletal Infection Society. Clin Orthop Relat Res. 2011, 469:2992-2994. 10.1007/s11999-011-2102-9

21. Marschall J, Evison J-M, Droz S, Studer UC, Zimmerli S: Disseminated tuberculosis following total knee arthroplasty in an HIV patient. Infection. 2008, 36:274-278. 10.1007/s15010-007-7011-1

22. Wang P-H, Shih K-S, Tsai C-C, Wang H-C: Pulmonary tuberculosis with delayed tuberculosis infection of total knee arthroplasty. J Formos Med Assoc. 2007, 106:82-85. 10.1016/S0929-6646(09)60221-7

23. Bryan WJ, Doherty JH, Sculco TP: Tuberculosis in a rheumatoid patient. a case report . Clin Orthop Relat Res. 1982, 171:206-208.

24. Tyrrell F, Stafford C, Yakrus M, Youngblood M, Hill A, Johnston S: Trends in testing for Mycobacterium tuberculosis complex From US Public Health Laboratories, 2009-2013. Public Health Rep. 2017, 132:56-64. 10.1177/0033354916679989

25. Bray C, Bell LN, Liang H, Haykal R, Kaiksow F, Mazza JJ, Yale SH: Erythrocyte sedimentation rate and Creactive protein measurements and their relevance in clinical medicine. WMJ. 2016, 115:317-321.

26. Kamada A, Amishima M: QuantiFERON-TB ${ }^{\circledR}$ Gold Plus as a potential tuberculosis treatment monitoring tool. Eur Respir J. 2017, 49:pii: 1601976. 10.1183/13993003.01976-2016

27. Mancuso JD, Bernardo J, Mazurek GH: The elusive "gold" standard for detecting Mycobacterium tuberculosis infection. Am J Respir Crit Care Med. 2013, 187:122-124. 10.1164/rccm.201211-2033ED

28. Pfyffer GE, Wittwer F: Incubation time of mycobacterial cultures: how long is long enough to issue a final negative report to the clinician?. J Clin Microbiol. 2012, 50:4188-4189. 10.1128/JCM.02283-12

29. Besser MI: Total knee replacement in unsuspected tuberculosis of the joint . Br Med J. 1980, 280:1434. 10.1136/bmj.280.6229.1434

30. Marmor M, Parnes N, Dekel S: Tuberculosis infection complicating total knee arthroplasty: report of 3 cases and review of the literature. J Arthroplasty. 2004, 19:397-400. 10.1016/j.arth.2003.10.015

31. Wray CC, Roy S: Arthroplasty in tuberculosis of the knee. Two cases of missed diagnosis . Acta Orthop Scand. 1987, 58:296-298.

32. Pulimood AB, Peter S, Rook GWA, Donoghue HD: In situ PCR for Mycobacterium tuberculosis in endoscopic mucosal biopsy specimens of intestinal tuberculosis and Crohn disease. Am J Clin Pathol. 2008, 129:846851. 10.1309/DKKECWQWMG4J23E3

33. Neogi DS, Kumar A, Yadav CS, Singh S: Delayed periprosthetic tuberculosis after total knee replacement: is conservative treatment possible?. Acta Orthop Belg. 2009, 75:136-140.

34. Nachiappan AC, Rahbar K, Shi X, et al.: Pulmonary tuberculosis: role of radiology in diagnosis and 
management. Radiographics. 2017, 37:52-72. 10.1148/rg.2017160032

35. Skoura E, Zumla A, Bomanji J: Imaging in tuberculosis. Int J Infect Dis. 2015, 32:87-93.

10.1016/j.ijid.2014.12.007

36. Harisinghani MG, McLoud TC, Shepard JA, Ko JP, Shroff MM, Mueller PR: Tuberculosis from head to toe. Radiographics. 2000, 20:449-470. 10.1148/radiographics.20.2.g00mc12449

37. Zeiger LS, Watters W, Sherk H: Scintigraphic detection of prosthetic joint and soft tissue sepsis secondary to tuberculosis. Clin Nucl Med. 1984, 9:638-639.

38. Spinner RJ, Sexton DJ, Goldner RD, Levin LS: Periprosthetic infections due to Mycobacterium tuberculosis in patients with no prior history of tuberculosis. J Arthroplasty. 1996, 11:217-222.

39. Nahid P, Dorman SE, Alipanah N, et al.: Official American Thoracic Society/Centers for Disease Control and Prevention/Infectious Diseases Society of America Clinical Practice Guidelines: treatment of drugsusceptible tuberculosis. Clin Infect Dis. 2016, 63:147-195. 10.1093/cid/ciw376

40. Kadakia AP, Williams R, Langkamer VG: Tuberculous infection in a total knee replacement performed for medial tibial plateau fracture: a case report. Acta Orthop Belg. 2007, 73:661-664.

41. Rowan FE, Donaldson MJ, Pietrzak JR, Haddad FS: The role of one-stage exchange for prosthetic joint infection. Curr Rev Musculoskelet Med. 2018, 11:370-379. 10.1007/s12178-018-9499-7

42. Hanssen AD: Managing the infected knee: as good as it gets . J Arthroplasty. 2002, 17:98-101. 10.1054/arth.2002.32458

43. Kunutsor SK, Whitehouse MR, Blom AW, et al.: One- and two-stage surgical revision of peri-prosthetic joint infection of the hip: a pooled individual participant data analysis of 44 cohort studies. Eur J Epidemiol. 2018, 33:933-946. 10.1007/s10654-018-0377-9

44. Khater FJ, Samnani IQ, Mehta JB, Moorman JP, Myers JW: Prosthetic joint infection by Mycobacterium tuberculosis: an unusual case report with literature review. South Med J. 2007, 100:66-69. 10.1097/01.smj.0000232972.50186.4c

45. Gottfriedsen TB, Schrøder HM, Odgaard A: Knee arthrodesis after failure of knee arthroplasty: a nationwide register-based study. J Bone Joint Surg Am. 2016, 98:1370-1377. 10.2106/JBJS.15.01363

46. Conway JD, Mont MA, Bezwada HP: Arthrodesis of the knee. J Bone Joint Surg Am. 2004, 86:835-848.

47. MacDonald JH, Agarwal S, Lorei MP, Johanson NA, Freiberg AA: Knee arthrodesis. J Am Acad Orthop Surg. 2006, 14:154-163.

48. Carrega G, Bartolacci V, Burastero G, Finocchio GC, Ronca A, Riccio G: Prosthetic joint infections due to Mycobacterium tuberculosis: a report of 5 cases. Int J Surg Case Rep. 2013, 4:178-181. 10.1016/j.ijscr.2012.11.011

49. Wu CH, Gray CF, Lee G-C: Arthrodesis should be strongly considered after failed two-stage reimplantation TKA. Clin Orthop Relat Res. 2014, 472:3295-3304. 10.1007/s11999-014-3482-4 